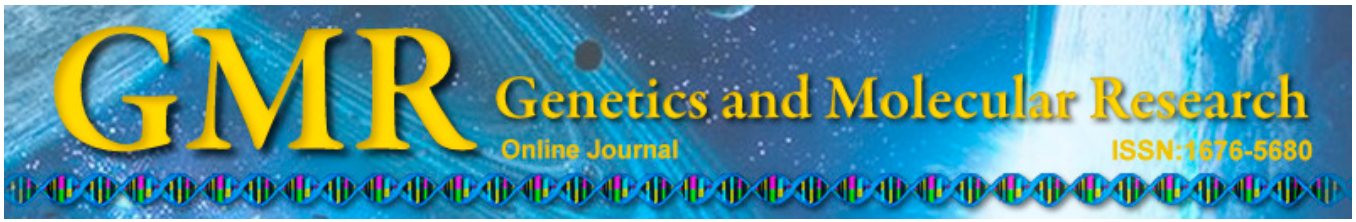

\title{
Early diagnosis of a Mexican variant of Papaya meleira virus (PMeV-Mx) by RT-PCR
}

\author{
E. Zamudio-Moreno ${ }^{1}$, J.H. Ramirez-Prado ${ }^{2}$, O.A. Moreno-Valenzuela ${ }^{1}$ \\ and L.A. Lopez-Ochoa ${ }^{1}$ \\ ${ }^{1}$ Unidad de Bioquímica y Biología Molecular de Plantas, \\ Centro de Investigación Científica de Yucatán, Mérida, Yucatán, México \\ ${ }^{2}$ Unidad de Biotecnología, Centro de Investigación Científica de Yucatán, \\ Mérida, Yucatán, México \\ Corresponding author: L.A. Lopez-Ochoa \\ E-mail: luisa_lopez@cicy.mx
}

Genet. Mol. Res. 14 (1): 1145-1154 (2015)

Received May 29, 2014

Accepted September 22, 2014

Published February 6, 2015

DOI http://dx.doi.org/10.4238/2015.February.6.18

\begin{abstract}
Papaya meleira disease was identified in Brazil in the 1980s. The disease is caused by a double-stranded RNA virus known as Papaya meleira virus (PMeV), which has also been recently reported in Mexico. However, previously reported $\mathrm{PMeV}$ primers failed to diagnose the Mexican form of the disease. A genomic approach was used to identify sequences of the Mexican virus isolate, referred here to as PMeV-Mx, to develop a diagnostic method. A mini cDNA library was generated using total RNA from the latex of fruits; this RNA was also sequenced using the Illumina platform. Sequences corresponding to the previously reported 669-base pair sequence for $\mathrm{PMeV}$ from Brazil (PMeV-Br) were identified within the PMeV-Mx genome, exhibiting 79-92\% identity with $\mathrm{PMeV}-\mathrm{Br}$. In addition, a new sequence of 1154-base pairs encoding a putative RNA-dependent RNA polymerase was identified in PMeV-Mx. Primers designed against this sequence detected both virus isolates, 2 amplicons of 173 and 491 base pairs from PMeV-Br and PMeV-Mx, and shared 100 and 98\% identity, respectively. PMeV-Mx was found in the latex of fruits, in seedlings,
\end{abstract}


and in the leaves, flowers, petioles, and seeds of mature plants. PMeV$\mathrm{Mx}$ was more abundant in the latex of fruits than in the leaves. The limit of detection of the CB38/CB39 primer pair was $1 \mathrm{fg}$ and $1 \mathrm{pg}$ using total RNA extracted from the latex of fruits and from seedlings, respectively. A sensitive and early diagnosis protocol was developed; this method will enable the certification of seeds and seedlings prior to transplantation to the field.

Key words: Double-stranded RNA virus; Latex; Papaya meleira virus; $\mathrm{PMeV}$; Reverse transcription-polymerase chain reaction detection; Virus variant

\section{INTRODUCTION}

Papaya meleira disease, also known as "sticky disease", is caused by a doublestranded RNA (dsRNA) virus that consists of 40-nm icosahedric particles called Papaya meleira virus (PMeV) (Kitajima et al., 1993; Zambolin et al., 2003). This is a unique plant virus because it is highly abundant in the laticifers of infected plants (Kitajima et al., 1993) that resists the harsh conditions of the latex and contains a dsRNA genome of approximately $12 \mathrm{~kb}$ (Zambolin et al., 2003). Although meleira severely affects papaya production in Brazil, very little is known about PMeV; additionally, it has not been sanctioned by the International Committee on Taxonomy of Viruses. Moreover, only a 669-base pair (bp) sequence encoding a fragment of a putative RNA-dependent RNA polymerase (RdRp) has been reported for this virus (Araújo et al., 2007).

Meleira disease was initially described in the early 1980s in Brazil (Kitajima et al., 1993), and was recently detected in Mexican orchards (Perez-Brito et al., 2012). The first symptom is necrosis of the leaf tips, followed by spontaneous exudation of watery-translucent latex in immature fruits. When this latex is oxidized, the fruit surface becomes darkened and the pulp is damaged, reducing the fruit's commercial value (Ventura et al., 2004). The removal and destruction of diseased plants is the only method available for controlling Papaya meleira disease (Lima et al., 2001; Ventura et al., 2004). Although the transmission mechanism has been examined in previous studies, it is not well understood (Rodrigues et al., 2009; Abreu et al., 2012).

A diagnostic assay based on extraction and visualization of nucleic acids from the latex of fruits separated on $0.7 \%$ agarose gels has been developed, in which the presence of a $12-\mathrm{kb}$ dsRNA band indicates presence of the disease (Tavares et al., 2004). This is performed in combination with an assessment of symptoms in the field, including the timing of latex crystallization upon immature fruit wounding, which is lower in diseased plants (Ventura et al., 2004). This method is fast, inexpensive, and reliable, and can be used to detect PMeV because the virus is abundant in the latex of fruits (Kitajima et al., 1993; Ventura et al., 2004). However, because symptoms typically do not appear until the papaya plant is flowering and producing fruits, the plants can only be diagnosed when they are approximately 6-9 months old. In 2007, a reverse transcription-polymerase chain reaction (RT-PCR) method based on the 669-bp partial sequence of PMeV was described (Araújo et al., 2007); however, this method is only suitable for detecting the virus in the latex of fruits (Abreu et al., 2012). Recently, internal primers directed against this sequence were designed, enabling the detection of the virus on leaves using conventional and quantitative RT-PCR (Abreu et al., 2012). 
In Mexico, similar symptoms to meleira disease were observed in papaya orchards, including in the Maradol variety, except for necrosis of the leaf tips. The presence of PMeV was determined using a protocol based on extraction and visualization of nucleic acids on agarose gels (Tavares et al., 2004), and a 12-kb band was observed in diseased plants (PerezBrito et al., 2012). Using nuclease treatments, it was shown that the 12-kb band was dsRNA. In addition, the disease was transmitted when the latex from diseased plants was injected into the apex of healthy plants (Perez-Brito et al., 2012). However, attempts to detect PMeV from Mexican orchards using the previously reported C05-3' primer failed (Araujo et al., 2007; Perez-Brito D, Tapia-Tussell R and Magaña A, personal communication and our own unpublished results). Thus, in this study, we developed an RT-PCR diagnostic method for the Mexican disease, which is sufficiently sensitive for detecting the virus not only in the latex of fruits but also in the leaves and other plant organs, to reduce the 6-9-month period that is currently required to achieve a positive diagnosis using RNA extracted from the latex of fruits. Our goal was to provide molecular evidence to support the findings of the first report of the presence of PMeV in Mexico (Perez-Brito et al., 2012).

\section{MATERIAL AND METHODS}

\section{Plant material and RNA extraction}

Tissues from a diseased mature papaya plant (cv. Maradol) growing in an orchard in Conkal, Yucatan, Mexico, were harvested, frozen in liquid nitrogen, and stored at $-80^{\circ} \mathrm{C}$ until use. For the seedling assays, seeds were germinated in peat moss substrate and incubated at $27^{\circ} \mathrm{C}$ under a $16 / 8$-h light/dark photoperiod; hypocotyls were injected with $20 \mu \mathrm{L}$ diseased latex diluted to $50 \%$ in phosphate buffer, $\mathrm{pH} 7.0$ (Abreu et al., 2012). The first 2 true leaves were collected at 12 days post-inoculation, frozen, and stored at $-80^{\circ} \mathrm{C}$ until use. Total RNA was isolated from $100 \mathrm{mg}$ frozen tissue using the RNeasy Plant mini kit (Qiagen, Hilden, Germany), including DNAse I treatment. The latex of fruits from diseased papaya plants was collected from 2 orchards located in Morelos, Quintana Roo, Mexico. Total RNA was extracted using the protocol of Tavares et al. (2004), and it was further purified using RNeasy columns (Qiagen). RNA was quantified using a NanoDrop Spectrophotometer (model 2000C, Thermo Scientific, Waltham, MA, USA).

\section{cDNA library construction and sequence analysis}

A mini cDNA library was constructed using random hexamers and reagents provided in the ProtoScript ${ }^{\circledR}$ M-MuLV First Strand cDNA Synthesis Kit following the manufacturer protocol (New England Biolabs, Ipswich, MA, USA) as well as $1 \mu \mathrm{g}$ total RNA extracted from the latex of fruits of 1 diseased papaya plant (cv. Maradol), which was obtained from an orchard at the state of Quintana Roo, Mexico. RNA primers were generated after treatment of the first-strand-RNA duplex with RNAse H. The Escherichia coli DNA polymerase I large fragment (Klenow) and dNTPs were added for second-strand synthesis, and the reaction was incubated at $16^{\circ} \mathrm{C}$ for $2.5 \mathrm{~h}$. Adenine ends were added with Taq polymerase (all reagents from New England Biolabs) and the cDNA was column-purified and ligated into the pGEM-T Easy vector (Promega, Madison, WI, USA). E. coli DH5 $\alpha$ competent cells were transformed and seeded on selective Luria-Bertani media. White colonies were selected for insert verifica- 
tion using PCR and restriction enzyme analysis. Plasmid DNA was sequenced at the Clemson University Genomics Institute (Clemson, SC, USA) using Big Dye terminators (Applied Biosystems, Foster City, CA, USA). Contigs were assembled using the Sequencher software version 5.2.3 (Gene Codes Corporation, Ann Arbor, MI, USA). Nucleotide sequences were compared against the National Center for Biotechnology Information (NCBI) databases using tBLASTx. Protein sequences were aligned using the Clustal W algorithm (Larkin et al., 2007) implemented in the "Geneious 6.1.2" software.

\section{Diagnosis by RT-PCR}

RT-PCRs were performed in 2 steps. For the first cDNA synthesis, 100 ng total RNA, extracted from the latex of fruits or from plant tissues, was mixed with either $1.5 \mu \mathrm{M}$ specific primers or an oligo-dT/random hexamer mix adjusted to $10 \mu \mathrm{L}$ with $\mathrm{ddH}_{2} \mathrm{O}$ and incubated at $70^{\circ} \mathrm{C}$ for $5 \mathrm{~min}$. Next, the RNA/primer mix was used in $20-\mu \mathrm{L}$ reactions containing $10 \mathrm{U}$ M-MuLV reverse transcriptase, 1X M-MuLV RT buffer, $0.25 \mathrm{mM}$ dNTPs, and $0.25 \mathrm{mM}$ dithiothreitol (all reagents from New England Biolabs). The reactions were incubated for $1 \mathrm{~h}$ at $42^{\circ} \mathrm{C}, 15 \mathrm{~min}$ at $70^{\circ} \mathrm{C}$, and adjusted to a final volume of $50 \mu \mathrm{L}$. For second-strand synthesis, $50 \mu \mathrm{L}$ PCRs contained $0.2 \mu \mathrm{M}$ specific primers (Table 1), $0.2 \mathrm{mM}$ dNTPs, $1.25 \mathrm{U}$ Taq polymerase, $1 \mathrm{X}$ Thermo Pol buffer, and $2 \mu \mathrm{L}$ first-strand cDNA. Reactions were performed using the following program: $94^{\circ} \mathrm{C}$ for $4 \mathrm{~min}, 30$ cycles of $30 \mathrm{~s}$ for $94^{\circ} \mathrm{C}, 52^{\circ}-60^{\circ} \mathrm{C}$ for $30 \mathrm{~s}, 72^{\circ} \mathrm{C}$ for $1 \mathrm{~min}$, and a 10 -min final extension at $72^{\circ} \mathrm{C}$. Next, $5 \mu \mathrm{L}$ was loaded onto a $0.8 \%$ agarose gel containing ethidium bromide. To assess the limit of detection (LOD), serial 10-fold dilutions of total RNA were used as a template in $25-\mu \mathrm{L}$ 1-step RT-PCRs. The PCR products were purified using the QIAquick PCR purification kit (Qiagen) and ligated into the pGEMT-easy vector. Plasmid DNA from 3 positive clones was sequenced as described above.

\begin{tabular}{|c|c|c|c|}
\hline Primer pair & Sequence & Size (bp) & $\operatorname{Tm}\left({ }^{\circ} \mathrm{C}\right)$ \\
\hline CB34 & 5' CCTCGAATGATTCAACATCGT 3' & 173 & 56.9 \\
\hline $\mathrm{CB} 35$ & 5' CCCCACATCTCACGTAAGATAC 3' & & \\
\hline CB36 & 5' GTACACAGTCCGTGGTCGACG 3' & 264 & 56.7 \\
\hline CB37 & 5' TGGCAGAACGAGATGCGCT 3' & & \\
\hline CB38 & 5' AGCGCATCTCGTTCTGCCA 3' & 491 & 60.2 \\
\hline CB39 & 5' GCCCGGATATACCGGCCT 3' & & \\
\hline Actin $\mathrm{FW}^{1}$ & 5' ATCTGCTGAACGGGAAATTG 3' & 300 & 60 \\
\hline Actin $\mathrm{RV}^{1}$ & 5' TGATGGCTGGAAGAGAACCT 3' & & \\
\hline
\end{tabular}

${ }^{1}$ Santos (2005).

\section{Illumina sequencing}

Total RNA extracted from the latex of fruits of a diseased plant was purified using the RNeasy Plant mini kit (Qiagen). cDNA library preparation and sequencing were performed at the Malaysia Genomics Resource Centre (Kuala Lumpur, Malaysia) using Illumina sequencing technology. Next, 100-bp paired-end reads were generated using the Illumina Hi Seq 2000 platform. prfectBLAST (Santiago-Sotelo and Ramirez-Prado, 2012) was used to create BLAST databases and perform sequence screening. 


\section{RESULTS}

\section{Cloning of a 1154-bp sequence from PMeV-Mx}

To generate an RT-PCR diagnostic method for the Mexican virus isolate, referred to as PMeV-Mx, it was necessary to first identify sequences that could serve as templates for RT-PCR. Total RNA extracted from the latex of fruits of diseased plants was used to build a mini cDNA library with the pGEM-T easy vector. A total of 134 positive colonies were identified and 6 were sequenced. Of the resulting sequences, 3 showed hits to RdRps from singlestranded RNA viruses (Table 2) in the NCBI databases and form a 1154-bp contig (Figure 1A). The closest identity of the predicted 384-amino acid partial protein is to RdRps from the unclassified Citrus yellow vein associated virus, followed by RdRps from umbraviruses (Table 2). The PMeV-Mx 1154-bp sequence was deposited into NCBI GenBank under accession No. KF781635.

Table 2. Amino acid percent identity of RdRp encoded by the 1154-bp contig.

\begin{tabular}{lcc}
\hline & Identity (\%) & Accession No. \\
\hline Citrus yellow vein associated virus (CYVV) & 42 & JX101610 \\
Carrot mottle mimic virus (CMoMV) & 39 & AAB81639.1 \\
Carrot mottle virus (CMoV) & 38 & ACJ03580.1 \\
Groundnut rosette virus (GRV) & 37 & NP_619714 \\
\hline
\end{tabular}

\section{A}

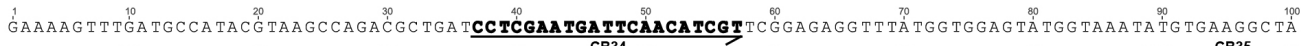

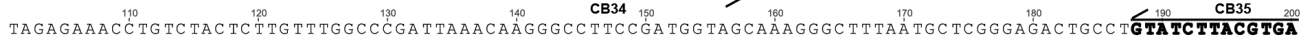

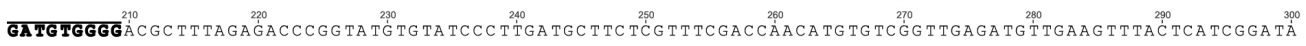

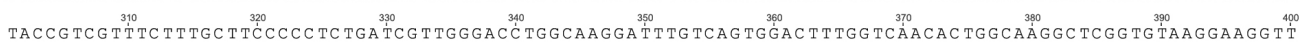

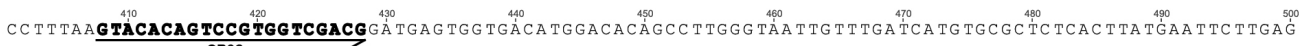

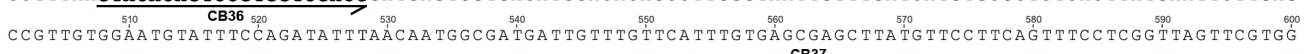

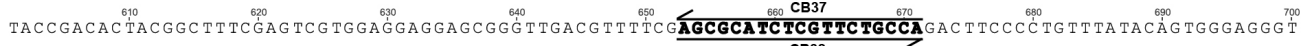

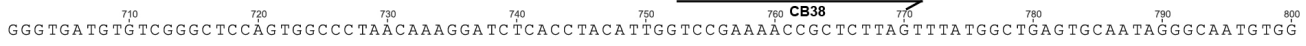

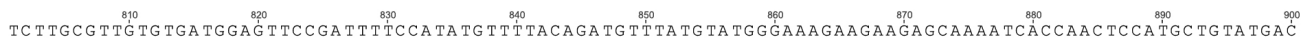

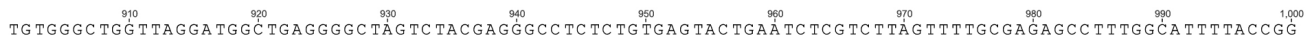

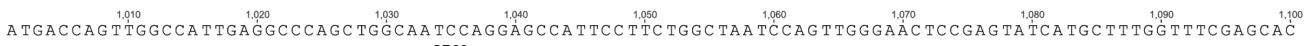

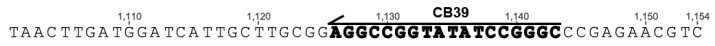

\section{B}

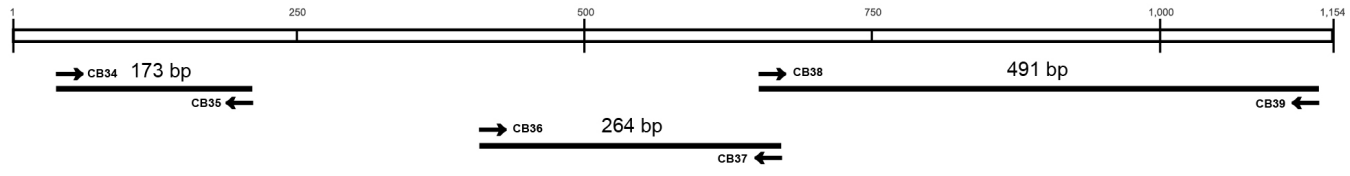

Figure 1. PMeV-Mx sequence and primers used for diagnosis by RT-PCR. A. Nucleotide sequence of the PMeV-Mx 1154-bp contig, primer-targeted sequences are indicated in bold letters. B. Schematic representation of amplicon positions in the 1154-bp contig, amplicon size is indicated. 


\section{RT-PCR diagnosis of PMeV-Mx using RNA from latex of fruits}

Six primers, 3 forward and 3 reverse, were designed from the 1154-bp contig to produce amplicons ranging from 173 to $491 \mathrm{bp}$ (Figure 1B and Table 1). Primer specificity was verified in silico using BLASTn. As shown in Figure 2A, the 3 primer pairs produced amplicons of the expected size in a latex sample from an infected plant from Mexico but not from a healthy plant. The specificity of the RT-PCR detection method was confirmed by cloning and sequencing the amplicons of 173 and $491 \mathrm{bp}$, produced using the primer pairs CB34/CB35 and CB38/CB39, respectively. To further test the RT-PCR protocol, the primer pair CB38/CB39 was selected for diagnosis of symptomatic and asymptomatic plants from 3 papaya orchards located in 2 states of the Yucatan Peninsula. PMeV-Mx was detected in all symptomatic plants (Figure 2B, lanes 5-9), as well as in 1 asymptomatic plant that tested negative using the 12-kb dsRNA band-visualization protocol (Figure 2B, lane 4), but not in healthy plants from different sources (Figure 2B, lanes 1 and 2). The LOD of this primer pair was $1 \mathrm{fg}$ (Figure 2C).
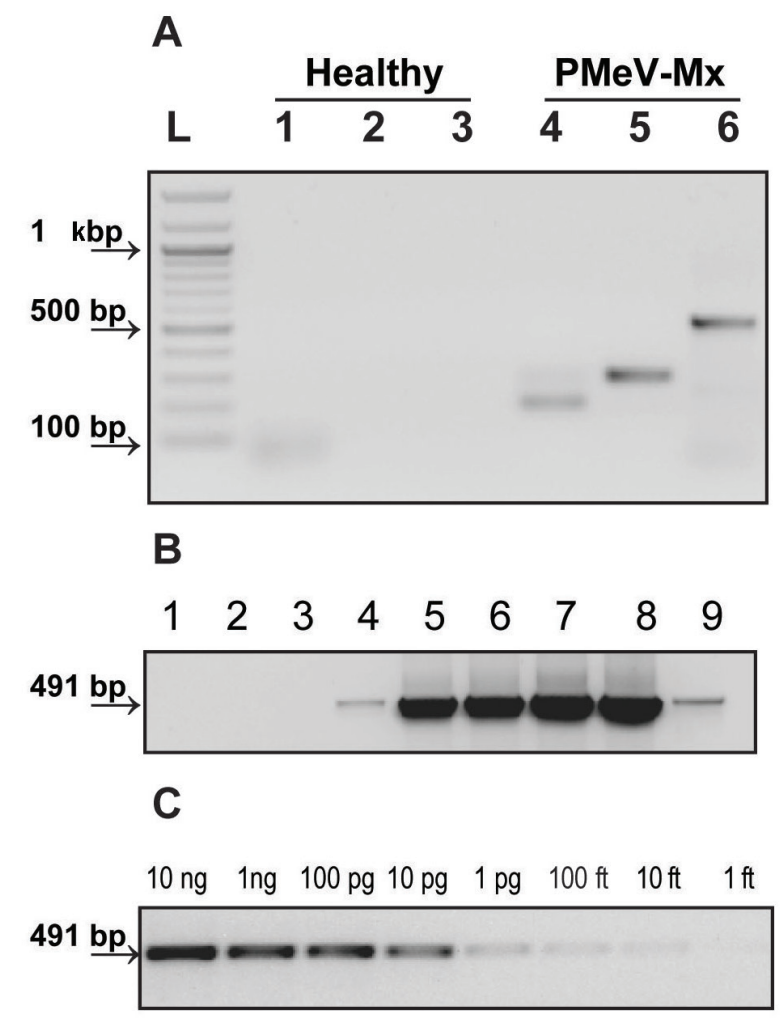

Figure 2. RT-PCR detection of PMeV-Mx in the latex of fruits. A. Primer pairs CB34/CB35 (lanes 1 and 4), CB36/ CB37 (lanes 2 and 5), and CB38/CB39 (lanes 3 and 6) targeting the 1154-bp contig were used with RNA from a healthy plant (left) and from an infected plant from Mexico (PMeV-Mx; right) to produce amplicons of 173, 264, and 491 bp, respectively. Lane $L=100$-bp DNA ladder (New England Biolabs). B. Primer pair CB38/CB39 was used for detection in asymptomatic (lanes 3 and 4) and symptomatic (lanes 5-9) papaya plants from different orchards; healthy plants served as a negative control (lanes 1 and 2). C. LOD of primer pair CB38/CB39. Amplicon size is indicated in $\mathrm{B}$ and $\mathrm{C}$. 


\section{RT-PCR diagnosis of PMeV-Mx in mature plants and seedlings}

To determine whether PMeV-Mx was present in the leaves as well as in other plant organs, RT-PCRs were performed using $100 \mathrm{ng}$ total RNA extracted from different organs of a mature diseased papaya plant and primer pair CB38/CB39. PMeV-Mx was present in young and mature leaves, flowers, petioles, and seeds, including the sarcotesta (Figure 3A, top), while no amplification was observed in leaves from a healthy plant. These results indicate that $\mathrm{PMeV}-\mathrm{Mx}$ is broadly distributed within the papaya plant. When primers for the papaya actin gene (Santos, 2005) were used for RNA quality control, equivalent amounts of papaya actin transcripts were observed in all organs (Figure 3A, bottom). To determine whether the RTPCR assay reported here was suitable for early diagnosis of PMeV-Mx, 8-day-old seedlings (cv. Maradol) in the expanded cotyledon stage were inoculated with diseased latex. PMeV-Mx was found in the latex-inoculated seedlings but not in mock-inoculated seedlings (Figure 3B, top); additionally, actin primers were used for RNA quality control (Figure 3B, bottom). These results demonstrate that the RT-PCR protocol is effective for early diagnosis of PMeV-Mx. The LOD was $1 \mathrm{pg}$ in seedlings (Figure 3C) and $100 \mathrm{fg}$ in mature plants (data not shown).

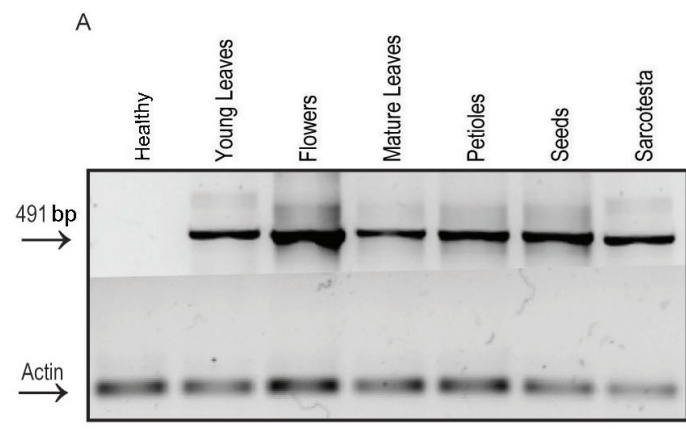

$\mathrm{B}$

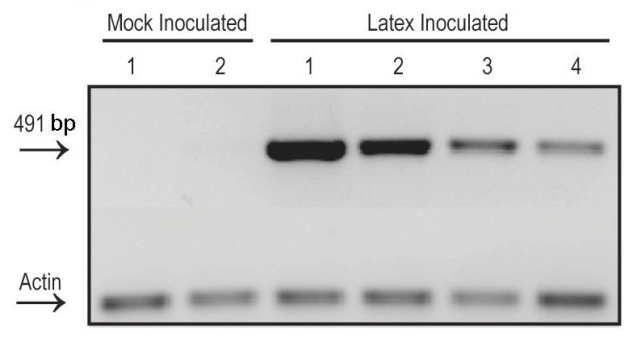

C

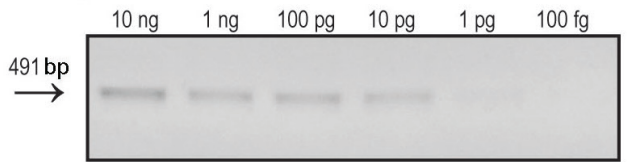

Figure 3. RT-PCR detection of PMeV-Mx in leaves and other plant tissues. Primer pair CB38/CB39 was used for RT-PCR in tissues of a mature diseased papaya plant (A), and true leaves from latex-inoculated seedlings (B). Actin primers were used for RNA quality control. (C) LOD of primer pair CB38/CB39 in leaves of a latex-inoculated seedling. The amplicon size is indicated (left). Lanes 1 and $2=$ RNA from two mocks; lanes $1-4=$ four PMeV-latex inoculated seedlings. 


\section{PMeV-Mx and PMeV-Br share the 1154- and 669-bp sequences}

Primers designed from the PMeV-Mx 1154-bp contig produced 3 amplicons of the expected size in $1 \mathrm{PMeV}-\mathrm{Br}$ sample (Figure 4A). The PMeV-Br PCR products of 173 and 491 bp were cloned and sequenced; they were found to share 100 and $98 \%$ identity to PMeV-Mx (data not shown). These amplicons target the $5^{\prime}$ and 3 ' ends of the 1154-bp contig, respectively (Figure 1B); therefore, these results suggest that $\mathrm{PMeV}-\mathrm{Br}$ contains the 1154-bp sequence identified in PMeV-Mx.
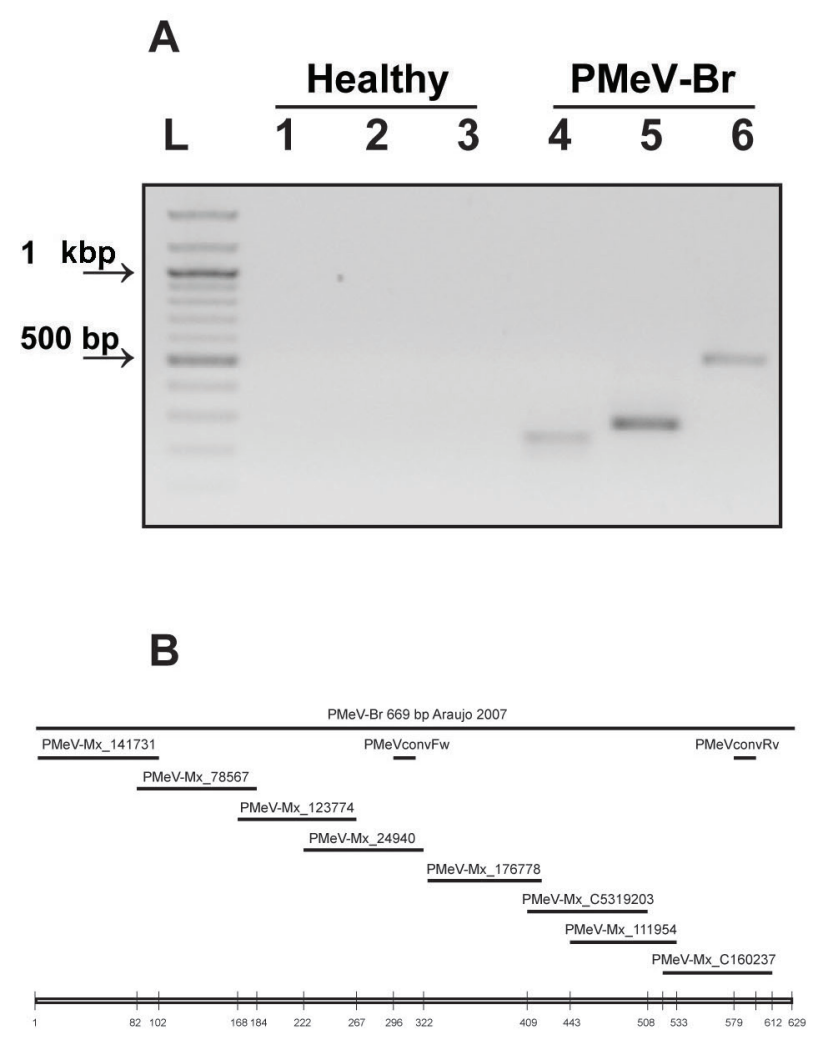

Figure 4. PMeV-Mx and PMeV-Br share the 1154-bp contig and the 669-bp sequence. A. Primer pairs CB34/CB35 (lanes 1 and 4), CB36/CB37 (lanes 2 and 5), and CB38/CB39 (lanes 3 and 6) targeting the 1154-bp contig were used with RNA extracted from the latex of fruits from a healthy plant (left) and from an infected plant from Brazil (PMeV-Br; right) to produce amplicons of 173, 264, and $491 \mathrm{bp}$, respectively. Lane $L=100$-bp DNA ladder (New England Biolabs). B. Schematic representation of the 669-bp sequence from PMeV-Br (Araújo et al., 2007) targeted by some of the PMeV-Mx Illumina reads. The binding sites of internal PMeVconv Fw and PMeVconv Rv primers (Abreu et al., 2012) are also indicated.

In contrast, the CO5-3' primer failed to produce the expected amplicon in different samples of papaya plants infected with PMeV-Mx (data not shown); this primer targets the 669-bp sequence of PMeV-Br (Araújo et al., 2007). To determine whether the PMeV-Mx genome contains sequences matching the 669-bp region, RNA from a PMeV-Mx diseased plant was sequenced using Illumina technology. Several reads spanned the PMeV-Br 669-bp 
sequence, forming 1 contig that targets positions 1-620 (Figure 4B). When aligned independently, these reads exhibited $79-92 \%$ identity to the $669-\mathrm{bp} \mathrm{PMeV-Br}$ sequence reported by Araújo et al. (2007) (no accession number available). These results reveal that PMeV-Mx contains the RdRp region encoded by the 669-bp sequence, but that this sequence has diverged in PMeV-Mx.

\section{DISCUSSION}

In this study, a 1154-bp contig from the PMeV-Mx genome was obtained, and based on this sequence, 3 primer pairs for RT-PCR diagnosis were designed. The primer pair CB38/ CB39 was the most efficient for PMeV-Mx detection in seedlings as well as in different tissues of a mature papaya plant, including seeds. The LOD of primer pair CB38/CB39 was $1 \mathrm{fg}$ in the latex of fruits and $1 \mathrm{pg}$ in the leaves of seedlings, indicating that $\mathrm{PMeV}-\mathrm{Mx}$ is more abundant in the latex of fruits than in the leaves. These results are consistent with previous reports for PMeV-Br (Zambolim et al., 2003; Tavares et al., 2004; Abreu et al., 2012). An early diagnostic assay for PMeV-Mx will be useful for farmers and local authorities, enabling the certification of seeds and seedlings prior to transplantation in the field and for the detection of disease in the field prior to fruit production. These methods will reduce the time required for diagnosis and economic losses.

The 3 primer pairs developed from the 1154-bp contig found in PMeV-Mx were also able to detect PMeV-Br. Amplicons of 173 and $491 \mathrm{bp}$ targeted the 5' and $3^{\prime}$ ends of the 1154bp contig, respectively, and shared sequence identity with PMeV-Mx. These results demonstrate that $\mathrm{PMeV}-\mathrm{Br}$ contains the 1154-bp contig. This sequence encodes and $\mathrm{RdRp}$, which showed closer similarity to RdRps from single-stranded RNA viruses (Table 2), including the umbravirus Ground nut rosette virus (GRV), while the 669-bp sequence was more similar to RdRps from dsRNA viruses (Araujo et al., 2007). However, when the 669-bp sequence was identified using RNA from purified viral particles, sequences encoding a protein with similarity to the polymerase from GRV were detected (Araujo et al., 2007). Using Illumina sequencing, reads showing similarity to the 669-bp sequence were also found in PMeV-Mx; however, their identity varied from $79-92 \%$. Furthermore, when internal PMeVconv primers (Abreu et al., 2012) were used with the PMeV-Mx RNA samples, 3 bands were produced after a second round of amplification; these bands shared similarity with the $\mathrm{PMeV}-\mathrm{Br}$ 669-bp sequence (Perez-Brito D, Tapia-Tussell R, and Magaña A, personal communication). These results demonstrate that PMeV-Mx also contains the RdRp region encoded by the 669-bp sequence. However, variation throughout the 669-bp sequence in PMeV-Mx suggests that this virus is a variant of PMeV. The presence of polymorphisms may explain the failure of the CO5-3' primer to produce the 669-bp amplicon in the PMeV-Mx samples. Primer discrimination by Taq polymerase failing to extend primer-3' ends with single-nucleotide changes is the basis of allele-specific PCR, which is applied to disease diagnosis and polymorphism analysis (Huang et al., 1992). Analysis of variation in $6 \mathrm{PMeV}$ isolates from different states in Brazil showed that 2 isolates, $\mathrm{PMeV}-2$ and $\mathrm{PMeV}-23$, exhibited 22 and $26 \%$ nucleotide divergence within the 669-bp sequence (Daltro et al., 2010). Recently, a larger study showed that $31 \mathrm{PMeV}$ isolates diverged by up to $12 \%$ in the same region (Daltro et al., 2014). These results provide a strong correlation for $\mathrm{PMeV}$ as the causal agent of the Mexican disease, and also provide molecular evidence to support the first report of PMeV in Mexico (Perez-Brito et al., 2012). In addition, the diagnostic method for PMeV-Mx and new cDNA sequence reported in this study will be 
useful for the early detection of the virus to reduce virus spread and economic losses. These findings will also be generally applicable to the study of meleira disease.

\section{ACKNOWLEDGMENTS}

The authors thank Daisy Perez-Brito, Raul Tapia-Tussel, and Annuar Magaña at GeMBio CICY for the PMeV-Mx samples; Eduardo Chumbino de Andrade at EMBRAPA Bahia, Brazil, for the latex sample; and Luis Carlos Gutierrez-Rodriguez for technical support. Research supported in part by funds obtained from Consejo Nacional de Ciencia y Tecnología (CONACYT, grant \#98920) to L.A. Lopez-Ochoa and a patent grant to Dr. Inocencio Higuera Ciapara, former Director of CICY. E. Zamudio-Moreno is indebted to CONACYT for her Ph. D. scholarship (\#175118).

\section{REFERENCES}

Abreu PM, Piccin JG, Rodrigues SP, Buss DS, et al. (2012). Molecular diagnosis of Papaya meleira virus (PMeV) from leaf samples of Carica papaya L. using conventional and real-time RT-PCR. J. Virol. Methods 180: 11-17.

Araujo MM, Tavares ET, Silva FR, Marinho VL, et al. (2007). Molecular detection of Papaya meleira virus in the latex of Carica papaya by RT-PCR. J. Virol. Methods 146: 305-310.

Daltro CB, dos Santos AF, Velame KV and de Andrade EC (2010). Análise Molecular de Isolados do Vírus da Meleira do Mamoeiro (Papaya meleira virus - PMeV). In: Anais do Congresso Brasileiro de Fruticultura Sociedade Brasileira de Fruticultura, Natal.

Daltro CB, Abreu EM, Aragão FL and Andrade EC (2014). Genetic diversity studies of Papaya meleira virus. Trop. Plant Pathol. 39: 104-108.

Huang MM, Arnheim N and Goodman MF (1992). Extension of base mispairs by Taq DNA polymerase: implications for single nucleotide discrimination in PCR. Nucleic Acids Res. 20: 4567-4573.

Kitajima E, Rodrigues C, Silveira J, Alves F, et al. (1993). Association of isometric virus-like particles, restricted to laticifers, with meleira (sticky disease) of papaya (Carica papaya). Fitopatol. Bras. 18: 118-122.

Larkin MA, Blackshields G, Brown NP, Chenna R, et al. (2007). Clustal W and Clustal X version 2.0. Bioinformatics 23: 2947-2948.

Lima RCA, Lima JAA, Souza Jr MT and Pio-Ribeiro G (2001). Etiologia e estratégias de controle de viroses do mamoeiro no Brasil. Fitopatol. Bras. 26: 689-702.

Perez-Brito D, Tapia-Tussell R, Cortes-Velazquez A and Quijano-Ramayo A (2012). First report of Papaya meleira virus (PMeV) in Mexico. Afr. J. Biotechnol. 11: 13564-13570.

Rodrigues SP, Andrade JS, Ventura JA and Lindsey GG (2009). Papaya meleira virus is neither transmitted by infection at wound sites nor by the whitefly Trialeurodes variabilis. J. Plant Pathol. 1: 87-91.

Santiago-Sotelo P and Ramirez-Prado JH (2012). prfectBLAST: a platform-independent portable front end for the command terminal BLAST+ stand-alone suite. Biotechniques 53: 299-300.

Santos MP (2005). Indução de Sistema de Defesa do Mamoeiro Como Resposta à Elicitores Químico (Óxido Nítrico) e Biológico (Saccharomyces cerevisiae). Master's thesis, Federal University of Espírito Santo, Vitória.

Tavares ET, Tatagiba JS, Ventura JA and Souza Jr MT (2004). Two new systems for early diagnosis of papaya sticky disease. Fitopatol. Bras. 29: 563-566.

Ventura JA, Costa H and Tatagiba JdS (2004). Papaya Diseases and Integrated Control. In: Diseases of Fruits and Vegetables: Diagnosis and Management (Naqvi SAMH, ed.). Klumer Academic Publishers, London, 201-268.

Zambolim E, Kunieda-Alonso S, Matsuoka K and De Carvalho (2003). Purification and some properties of Papaya meleira virus, a novel virus infecting papayas in Brazil. Plant Pathol. 52: 389-394. 